\title{
Model-Based EELS Quantification and ELNES Phase Mapping Using Experimentally Measured Cross-Sections
}

\author{
Bernhard Schaffer ${ }^{1}$, Liam Spillane ${ }^{1}$ and Paul J. Thomas ${ }^{1 *}$ \\ 1. Gatan Inc., Pleasanton, CA, USA \\ * Corresponding author: pthomas@gatan.com
}

Electron-energy-loss spectroscopy (EELS) can reveal a wealth of information about the sample under investigation with high sensitivity and high spatial resolution. However, extracting this information in an optimal manner is often non-trivial for a variety of reasons. In recent years, model-based quantification has both simplified and improved the accuracy of the quantification process in EELS (e.g. [1, 2]). It could be shown that using a combination of a power-law decaying background along with theoretically computed cross-sections as first-order approximation of the expected edge shape gives a reasonably robust way of performing compositional quantification, automatically separating out contributions of overlapping edge shapes in many commonly encountered situations. Provided a suitable EELS low-loss spectrum is available, plural scattering effects can also be incorporated into such an analysis to further improve the quantification. While this approach can greatly improve the reproducibility and accuracy of quantification and allows for very fast map computations in the spectrum-imaging application, some groups of tightly overlapping edges can still not be sufficiently separated automatically. This inability is caused by the theoretical cross-sections not correctly reproducing the electron loss near-edge finestructure (ELNES) features that are present in experimentally measured spectra.

The improved quantification scheme presented here utilizes experimentally measured cross-sections to improve the situation. The cross-section shape is readily derived from background-subtracted spectra, ideally corrected for plural scattering effects, to create an edge 'standard'. This edge standard can often (and ideally) be obtained directly from the spectrum-image data to be analyzed itself. Scaling of the standard is then either achieved by normalization with theoretically computed cross-sections, or it can be derived from the a-priori knowledge of the sample composition and thickness.

This new approach combines the advantages of the multiple linear least-square (MLLS) fitting of static reference spectra with the model-based quantification scheme. MLLS fitting of pre-measured standards has been shown previously to yield superior quantification compared to conventional background subtraction [3]. It can also achieve clean separation of edges for complex overlapping scenarios and incorporates plural scattering correction at the same time, yielding more accurate maps for specimens with thickness variations across the data (Fig.1). This is achieved in a simple, unified workflow. Furthermore, by allowing multiple concurrent experimental cross-section references to be specified for each individual core-loss edge, ELNES finger-printing can be incorporated into the same quantification routine (Fig. 2). This allows the separation of elements of different chemical states to be performed routinely, with plural scattering correction applied, as part of the regular elemental mapping process. This approach will be described, along with practical examples of common application use cases.

\section{References:}

[1] J Verbeeck et al., Ultramicroscopy 106 (2006), p. 11.

[2] PJ Thomas et al., Microsc. Microanal. 18 (S2) (2012), p. 968. 
[3] K Riegler et al., Ultramicroscopy 110 (2010), p. 8.

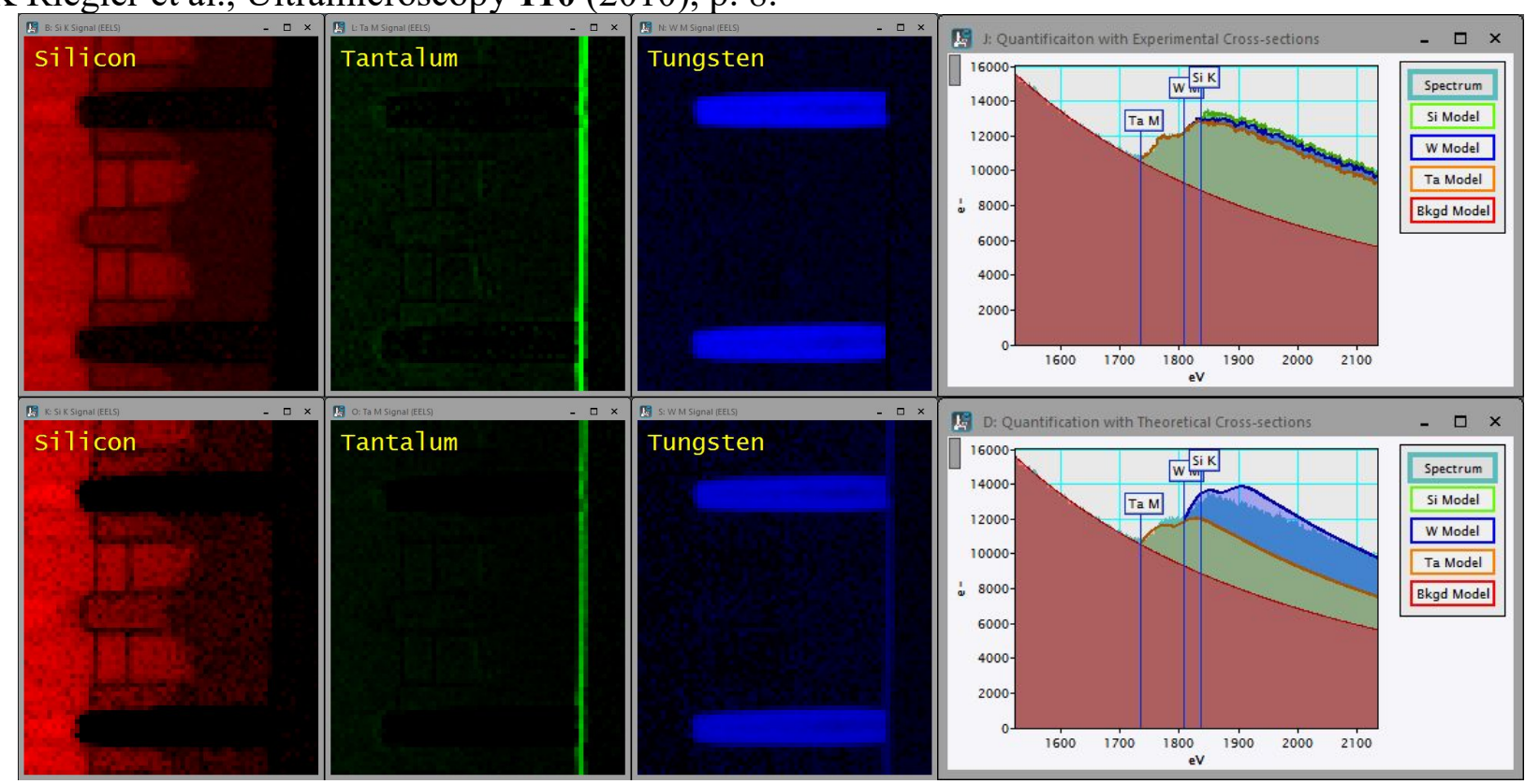

Figure 1. Semi-conductor device. Comparison of elemental intensity maps and quantified area-spectrum (2x30 SI pixels) computed by model-based quantification using experimental (top row) and theoretical (bottom row) cross-sections. The overlap of W M and Si K edge cannot be separated cleanly using only the theoretical Hartree-Slater cross-sections plus plural scattering convolution. Experimental crosssections taken from the data itself and scaled by theoretical cross-sections provide this separation.

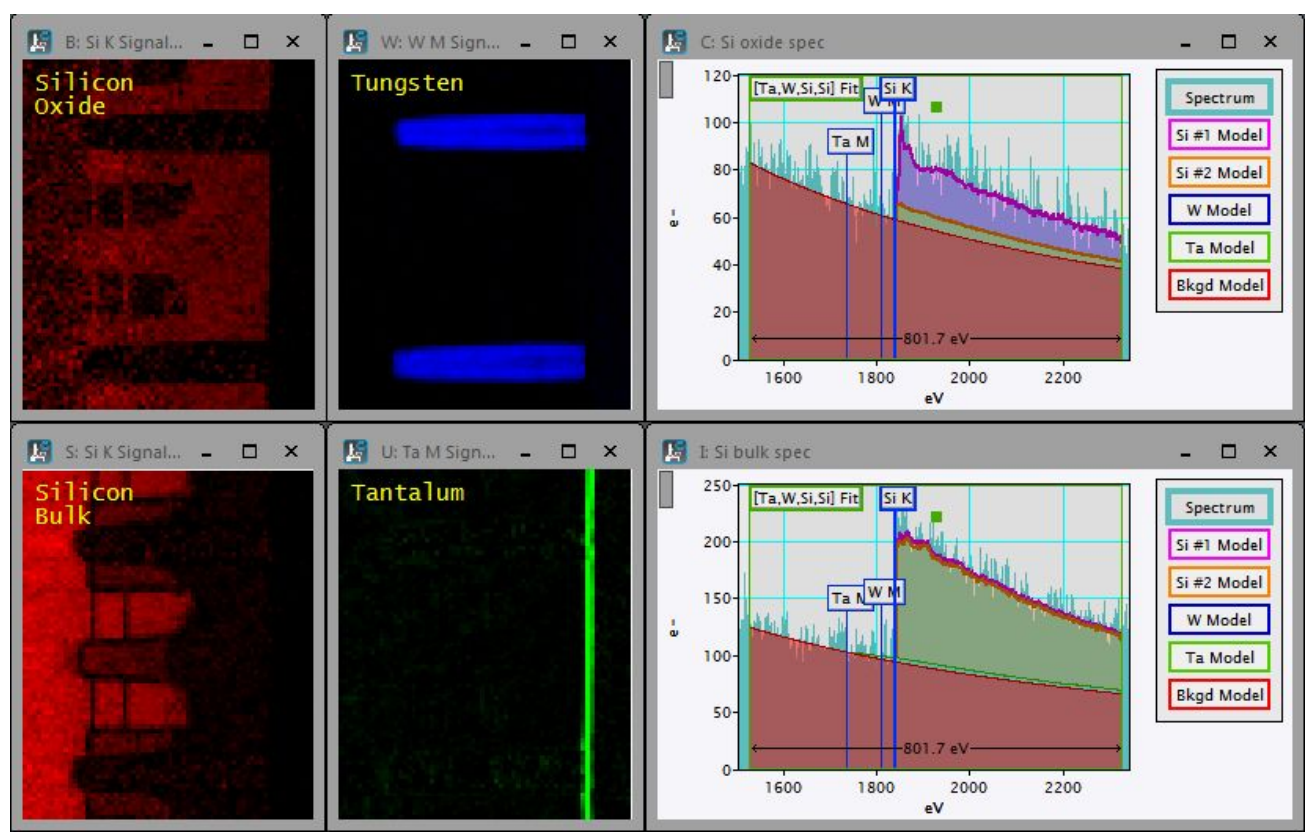

Figure 2. Same source data as Fig.1 above. ELNES finger-printing by providing multiple concurring experimental cross-sections during quantification. Elemental intensity maps and quantified point-spectra from silicon bulk and oxide regions are shown, respectively. 